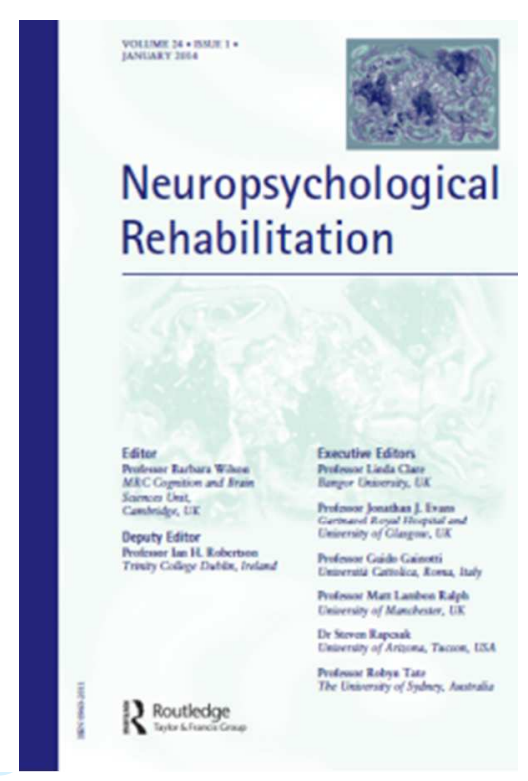

\title{
Reducing anger outbursts after a severe TBI: A single-case study
}

\begin{tabular}{|r|l|}
\hline Journal: & Neuropsychological Rehabilitation \\
\hline Manuscript ID & NRH-OA 130.15.R2 \\
\hline Manuscript Type: & Original Articles \\
\hline Complete List of Authors: & $\begin{array}{r}\text { Rochat, Lucien; University of Geneva, Cognitive Psychopathology and } \\
\text { Neuropsychology Unit } \\
\text { Manolov, Rumen; University of Barcelona, Behavioral Sciences Methods; } \\
\text { ESADE, Operations Management and Innovation } \\
\text { Aboulafia Brakha, Tatiana; Geneva University Hospitals, Neurology; } \\
\text { Geneva University Hospitals, Neurorehabilitation } \\
\text { Berner-Burkard, Christina; University of Geneva, Cognitive } \\
\text { Psychopathology and Neuropsychology Unit } \\
\text { Van der Linden, Martial; University of Geneva, Cognitive Psychopathology } \\
\text { and Neuropsychology Unit, }\end{array}$ \\
\hline Keywords: & $\begin{array}{l}\text { traumatic brain injury, anger, single-case, emotion recognition, } \\
\text { implementation intentions }\end{array}$ \\
\hline \multicolumn{2}{|c}{} \\
\hline
\end{tabular}

\section{SCHOLARONE \\ Manuscripts}




\section{Reducing anger outbursts after a severe TBI: A single-case study}

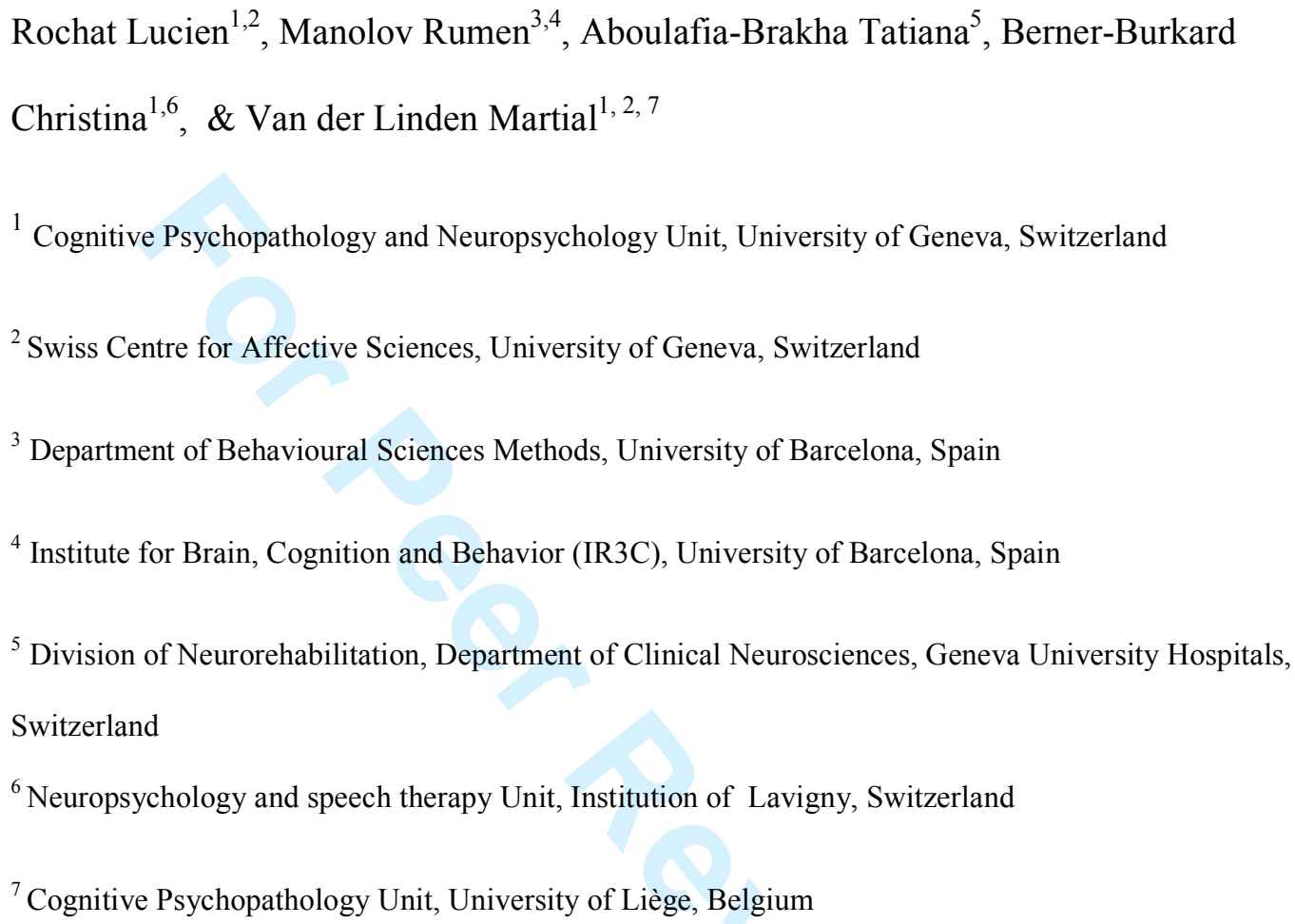




\begin{abstract}
Anger outbursts constitute a frequent behavioural issue after a traumatic brain injury (TBI) and have a strong negative impact on the social outcomes resulting from the TBI. However, few studies have examined the efficacy of specific intervention strategies to reduce the frequency and intensity of anger outbursts. We therefore performed a singlecase study on this topic by administering two successive and complementary psychological interventions with an $\mathrm{AB}$ design with maintenance (first intervention) and an AC design with maintenance plus a 1-month follow-up (second intervention) to a patient with a severe TBI. Whereas the first intervention focused on improving the recognition and expression of basic emotions, the second consisted of a self-regulation programme, including various features such as psychoeducation about self-control strategies, relaxation and assertiveness training that aimed to establish adequate behaviours, which were further promoted by an implementation intentions strategy in the patient's daily life. The results indicated that all interventions resulted in a reduced frequency and intensity of anger outbursts, and the data upheld the specificity of these effects. In addition, a meta-analytic integration of the effects of both interventions on the outcomes indicated a medium effect size. Further research is needed on other patients who experience long-standing anger outbursts to examine whether the observed gains can be replicated, sustained on a longer-term basis and improved.
\end{abstract}

Keywords: traumatic brain injury, anger, single-case, emotion recognition, implementation intentions. 


\section{INTRODUCTION}

Anger outbursts constitute a frequent behavioural issue after a traumatic brain injury (TBI). Indeed, between $12 \%$ and $76 \%$ of persons with TBI experience symptoms ranging from irritability to aggressive outbursts (e.g., Baguley, Cooper, \& Felmingham, 2006; Kim, Manes, Kosier, Baruah, \& Robinson, 1999; Tateno, Jorge, \& Robinson, 2003). In addition, these problematic behaviours are related to negative social outcomes such as family problems, social isolation and/or loss of employment (e.g., Kim et al., 2007).

Many studies have suggested the possibility of improving anger management and associated problems in persons with brain injury. In particular, cognitive behaviour therapy (CBT) has been shown to successfully decrease irritability and aggression in persons with TBI (e.g., Aboulafia-Brakha, Greber Buschbeck, Rochat, \& Annoni, 2013; Alderman, 2003; Medd \& Tate, 2000; Walker et al., 2010). However, besides focusing on the identification and modification of cognitive distortions that contribute to the maintenance of these problems, as well as developing adapted coping strategies as in a CBT approach (Giles \& Manchester, 2006), interventions should also focus on complementary variables that may contribute to the development and/or maintenance of maladapted behaviours, such as emotion expression and recognition. Indeed, the ability to decode and respond to emotions expressed by persons we are interacting with is a critical component for developing and maintaining harmonious social interactions (e.g., Driscoll, Dal Monte, \& Grafman, 2011). A meta-analysis performed on 296 adults with moderate to severe TBI indicated that patients significantly differ from matched controls in their ability to decode static facial expressions, the results showing a large effect size ( $d=1.1$; Babbage et al., 2011). Of note is that these deficits in emotion perception in patients with TBI have been associated with behavioural problems, lack of 
self-awareness, poor social competence and poorer social outcomes (Knox \& Douglas, 2009; Milders, Ietswaart, Crawford, \& Currie, 2008; Spikman et al., 2013; Watts \& Douglas, 2006). In addition, group-controlled randomised studies showed that intervention programmes designed to improve emotion recognition (by using errorless learning, imitation, vanishing cues) are successful, although the transfer of these effects into patients' daily lives has not been systematically established (Bornhofen \& McDonald, 2008b; Neumann, Babbage, Zupan, \& Willer, 2015; Radice-Neumann, Zupan, Tomita, \& Willer, 2009). One explanation for these poor transfer effects may first lie in the fact that emotion recognition impairments are associated with many variables (e.g., slowed processing speed, poor flexibility, or reduced selective or sustained attention; Ietswaart, Milders, Crawford, Currie, \& Scott, 2008; Mancuso et al., 2015; McDonald \& Saunders, 2005). Second, problematic behaviours may be underlain by a host of deficits or variables that these programmes do not act on (Bornhofen $\&$ McDonald, 2008a). Consequently, a complementary approach to the improvement of emotion recognition and expression is to provide patients with psychoeducation about self-regulation strategies, relaxation with deep breathing, and assertiveness training that will help them to decrease emotional overreactions and to develop more appropriate behaviours (see Demark \& Gemeinhardt, 2002). In this context, a specific selfregulation strategy called "implementation intentions" might be used to promote or facilitate the transfer of learning in the patient's daily life, especially in at-risk situations.

Numerous studies have shown that the execution of goal-directed behaviours can be enhanced by the use of implementation intentions (Gollwitzer, 2014). Implementation intentions are defined as a planning strategy that consists of specifying with as much detail as possible when, where and how one will perform a specific action 
(Gollwitzer, 1993). Such a plan is formulated as an if-then contingency by using the following structure: "If situation X arises, then I will perform behaviour Y" (e.g., "If I do not agree with my colleagues, then I will express my disagreement in a calm voice"). In such a plan, the "if" component specifies a suitable occasion for the implementation, whereas the "then" component identifies an adequate response to these target stimuli, a behaviour that allows one to reach one's goal (e.g., to behave in a socially adapted manner). Implementation intentions can also include a visual component, consisting of visualising oneself performing the intended action in the targeted situation. The imagery component may help to strengthen the encoding of the implementation intention (Cohen \& Gollwitzer, 2008).

This self-regulatory planning strategy has been shown to be effective in improving a wide range of cognitive functions and goal-directed behaviours. Indeed, in a meta-analysis examining the efficacy of implementation intentions, Gollwitzer and Sheeran (2006) showed a moderate to strong effect size $(d=0.65)$ for participants from the community and a large effect size in clinical samples $(d=1.10)$. According to Gollwitzer (1999), implementation intentions promote high activation of the specified situational cue (by perceptual, attentional and mnemonic processes) and create a link between the situation and the behaviour, so that when the situation occurs, the behaviour is automatically initiated (see also Brandstätter, Lengfelder, \& Gollwitzer, 2001; Lengfelder \& Gollwitzer, 2001; Wieber, Thürmer, \& Gollwitzer, 2015). Because several pieces of empirical evidence uphold the automaticity of implementation intentions, participants with a TBI who frequently present difficulties with controlled processes could benefit from this strategy. To the best of our knowledge, no studies to date have used implementation intentions (within a broader self-regulation programme that includes other features such as psychoeducation about self-regulation strategies, 
relaxation and assertive training) to reduce the frequency and intensity of behavioural problems such as anger outbursts after a TBI.

The objectives of the current study were to examine the efficacy of two successive psychological interventions that aimed to decrease both the frequency and the intensity of anger outbursts in a patient with a severe TBI. The first intervention focused on improving the recognition and expression of basic emotions, as this ability constitutes a central component for developing and maintaining harmonious social interactions.

However, this intervention does not provide the patient with a specific self-regulation strategy that could be used in at-risk situations. Therefore, the second and complementary intervention consisted of a self-regulation programme, including various features such as discussion about emotion regulation strategies, relaxation and assertiveness training that aimed to establish adequate behaviours, which were further promoted by an implementation intentions strategy in the patient's daily life.

This study illustrates an attempt to separate out and evaluate some of the components (emotion recognition vs. self-regulation) of a combination treatment for anger management after a TBI. This multicomponential approach to anger management after a TBI fits well with recommendations by authorities in the field (see Demark \& Gemeinhardt, 2002).

\section{CASE STUDY}

\section{Background and clinical history}

MD is a 47-year-old right-handed male who sustained a severe TBI after a 4-m fall in 2011. Magnetic resonance imaging revealed several haematomas (right frontal epidural and subdural, left frontal), a left anterior subarachnoid haemorrhage, bilateral fronto-basal haemorrhagic contusions and several fractures of the skull. 
Three months after the accident, MD was transferred to a neurological rehabilitation centre, where he benefited from several neuropsychological, ergotherapeutic and physiotherapeutic treatments. One year after the TBI, he also received a group intervention based on a CBT approach to decrease aggression and anger outbursts (focusing on anger awareness, self-monitoring, management of emotions in emergency situations, cognitive restructuring and prevention strategies; see Aboulafia-Brakha et al., 2013). The patient and his spouse stated that he benefited from this intervention in that he acquired more introspection into his difficulties. However, 1 year after the intervention, issues with anger outbursts remained, and MD was motivated to further improve his control of anger. Medical records showed that MD had no history of mental health or neurological difficulties before the TBI. At the time of assessment, a suspicion of obstructive sleep apnoea was mentioned and further diagnosed. A positive airway pressure device was thus introduced 2 weeks after the end of the first intervention and 8 weeks before starting the second intervention (see below).

MD spontaneously described frequent irritability and difficulty in managing anger since his brain injury. He pointed out that he had frequent anger outbursts when he was upset. He expressed his anger by yelling loudly, especially at home during interactions with his children when he felt that they lacked respect toward him, when they did not listen to him, or more generally when he did not agree with their behaviours or wishes. He also tended to ruminate on these angry episodes, wondering whether it was his fault or why he was so angry at the moment, which frequently prevented him from falling asleep at night. He reported symptoms of fatigue, low self-esteem and anxiety (e.g., he worried about his future and whether he might or might not improve at the cognitive and behavioural levels). 
According to his wife, the number of anger outbursts at home was on average four to five episodes weekly. During a prototypical anger outburst, MD shouted loudly, became agitated and did not let the interlocutor speak. She also reported that her husband became impulsive after the TBI, whereas she evoked an image of him having a reserved personality before the accident. When more specifically questioned, however, she mentioned that even before the TBI, her husband could become intent upon doing things his own way in order to ensure that his high standards were met, which could lead to some irritability, but anger outbursts were rare. She also reported that the relationship between MD and his children had become conflictual since the injury and that they had lost respect for their father. She also described symptoms of fatigue, especially when he returns home after work, low self-esteem, and worries about the future. In addition, she spoke of increased sadness because MD did not meet his parents as frequently as he used to do.

\section{Neuropsychological assessment}

A neuropsychological assessment performed 18 months after the TBI showed moderate impairments in executive functions (problem resolution integrating several constraints, inhibition), light to moderate deficits in long-term memory and slowing of reaction times. In a second assessment performed 3 years after the TBI in March 2014 before the intervention began, the deficits were globally identical (see Table 1).

\section{--INSERT TABLE 1 ABOUT HERE --}

The assessment emphasised cognitive impairments in executive functions (mental flexibility, divided attention, updating in working memory, planning and problem resolution), attention (slowing down of processing speed) and non-verbal episodic 
memory, which are compatible with the TBI (Table 1). In addition, difficulties in recognising emotions from static faces were stressed, in particular fear, and to a lesser extent anger and sadness, in the absence of general perceptive or semantic impairments.

\section{Rationale for the intervention}

Anger outbursts following MD's TBI could be associated with several variables, such as (1) impairments in emotion recognition and expression; (2) poor executive/attentional functioning; and (3) fatigue, which has also been associated with decreased self-regulation abilities (e.g., Belmont, Agar, \& Azouvi, 2009). Consequently, complementary succeeding interventions that specifically target or bypass these impairments were implemented in MD.

To decrease both the frequency and the intensity of anger outbursts, rehabilitation efforts first focused on improving emotion expression and recognition by means of several strategies, such as vanishing cues that enabled MD to direct his attention on relevant features of faces. This strategy was used because eye-tracking data during the baseline phase (see Table 5) revealed that MD's gaze focused much more on the mouth than on the area of the eyes when determining the emotion expressed by a face. The intervention then focused on physiological reactions related to each basic emotion and on imitation in a mirror, which provided MD with internal signals that could help him not only to better understand his own emotional reactions, but also to better recognise others' emotions.

The second intervention contains various features that aimed to stop the rise in anger and replace his habitual response (i.e., anger outbursts) with an assertive, socially adapted behaviour. For this purpose, various emotion regulation strategies were discussed, and relaxation training and assertiveness learning were performed in order to 
formulate an alternative behaviour that could be used by MD in stressful situations. Implementation intentions were then selected to promote the use of this alternative adapted behaviour in MD's everyday life, as they rely principally on automatic processes rather than on controlled mechanisms, which are impaired in MD.

In both interventions, repeated exercises, constant feedback and role playing were used to promote their transfer to daily life. Finally, independently of our interventions, a positive airway pressure device was successfully introduced to treat the obstructive sleep apnoea between the two interventions to decrease symptoms of fatigue.

\section{Measures of anger outbursts and other behaviours}

Anger outbursts were assessed daily with a home-made diary in which the spouse and the patient were instructed to place a check mark each time MD presented a target behaviour and to assess its intensity on a 10-point Likert scale from 1 (irritability) to 10 (strong outburst with physical contacts). Scores of frequency and intensity were averaged for each week. The target behaviours included various emotional reactions or behaviours such as exaggerated irritability, shouting, yelling, sudden movements, and verbal or physical aggression toward another person. Internalized anger (e.g., selfdirected bitterness and resentment) was not considered a target behaviour. In addition, in the same diary, both the patient and his spouse indicated the intensity of perceived physical fatigue on a 10-point Likert scale from 1 (slight fatigue) to 10 (strong fatigue), a control variable for which we did not expect any changes that resulted from our interventions (i.e., emotion recognition and expression and self-regulation). Note that the patient and the spouse each had their own diary. These behaviours were continuously assessed throughout both interventions. However, only the spouse's diary was taken into account in the analysis, as MD reported only one or two anger outbursts 
per week (generally the most intense episodes) or sometimes forgot to complete the schedule or to bring it back to the sessions.

In addition, the Neuropsychiatric Inventory (NPI; Cummings et al., 1994) and two questionnaires, the Dysexecutive Questionnaire (DEX; Wilson, Alderman, Burgess, Emslie, \& Evans, 1996) and the short version of the UPPS Impulsive Behavior Scale (Rochat et al., 2010), completed by MD's spouse, were used to assess whether the effect of the interventions generalised to other behaviours. These questionnaires were administered twice during the baseline and once during the post-test of each intervention.

The NPI consists of screening questions to determine whether behaviour changed after the TBI and whether the altered behaviour was present at the time of the interview (“in the current week"). It contains 12 subscales assessing delusions, hallucinations, agitation/aggression, dysphoria/depressed mood, anxiety, euphoria, apathy, disinhibition/impulsivity, irritability/lability, aberrant motor behaviour, nighttime disturbances and appetite/eating disturbances. If the relative responds positively to a screening question, the symptom is explored by means of several sub-questions focused on specific features of each disturbance. Questions about the symptom's severity, ranging from 1 (mild) to 3 (severe), and frequency, from 1 (rarely) to 4 (very frequently), are then completed by the relative. The score for each behavioural domain is the product of the frequency and severity subscore for that particular behaviour (maximum 12), with higher scores indicating greater emotional or behavioural difficulties.

The DEX contains 20 items measuring difficulties in everyday life as a result of executive dysfunction at a cognitive, emotional and behavioural level, with higher scores indicating greater difficulties. 
The short version of the UPPS Impulsive Behavior Scale (Rochat et al., 2010) assesses the multidimensional aspects of impulsivity after a TBI. The informants' version of this scale contains 16 items evaluating the four components of impulsivity, namely, urgency (e.g., "In the heat of an argument, he will say things that he later regrets"); (lack of) premeditation (e.g., "Before making up his mind, he considers all the advantages and disadvantages"); (lack of) perseverance (e.g., "Once he starts a project, he finishes it"); and sensation seeking (e.g., "He quite enjoys taking risks"). A current score for each dimension is computed, ranging from 4 to 16 . Higher scores indicate greater impulsivity.

\section{Measures of emotion recognition}

During the baseline phase, the Ekman 60 Emotional Faces Test (Ekman \& Friesen, 1976) was administered on three occasions to ensure stability of responses. This test was also administered once at the post-test. As MD's impairments in emotion recognition could have been secondary to his limited attentional and executive abilities (e.g., difficulties in selecting specific face areas that are relevant for emotion recognition or continuously maintaining attention in these areas to gather relevant information to determine the emotion expressed by the face), the test was also repeated on the third assessment with an eye-tracker device. A Tobii T120 Eye Tracker with a 17-inch thin-film transistor monitor (Tobii, Stockholm, Sweden; www.tobii.com) was used to assess visual exploration of the stimuli. The percentage of fixations in two relevant areas of the faces (around the mouth and around the eyes, including the eyebrows) was recorded. Indeed, the eyes and mouth constitute relevant facial features by which one can recognise emotions expressed by an interlocutor. In addition, the influence of practice effects from multiple pre-assessments was addressed by using 
another battery of emotional faces at the post-test: the Karolinska Directed Emotional Faces (KDEF; Lundqvist, Flykt, \& Öhman, 1998).

To explore any transference of learning from one modality (visual) to another modality (auditory) that MD had not been specifically trained for in the programme, we assessed vocal emotion recognition by using the vocal stimuli of the Emotion Recognition Index (Scherer \& Scherer, 2011). In this task, vocal portrayals of joy/happiness, sadness, fear, anger and neutral were played by four professional actors. Actors were asked to produce two "standard utterances" for their portrayals - "Hat sundig pron you ventsy" and "Fee gott laish jonkill gosterr" - to avoid any confounding effect due to semantics. The task includes 30 stimuli (anger: seven; fear: five; joy/happiness: six; sadness: eight; neutral: four). This task was administered on three occasions to ensure stability of responses during the baseline, as well as once in the post-test.

\section{Design}

The study took place over a 1-year period. After MD completed the first intervention, there was a 3-month interval, after which he completed the second intervention. For the first intervention, an $\mathrm{AB}$ design with maintenance was used, while for the second intervention, an AC design with maintenance plus a 1-month follow-up was applied. Two main outcomes (target behaviours) were assessed per intervention: the frequency and the intensity of anger outbursts (Figure 1).

\section{--INSERT FIGURE 1 ABOUT HERE-}

As it constitutes an important issue for MD, subjective physical fatigue was also measured. However, because fatigue was likely due to obstructive sleep apnoea, we 
expected no changes or only minor changes for this variable after the two interventions.

Rather, we expected symptoms of fatigue to decrease with the introduction of the continuous positive airway pressure (CPAP) device.

After a 1-month baseline measurement for each intervention (A), the intervention began (B or C). One 60- to 90-minute session per week was delivered face to face for 2 months, followed by a 1-month post-test. After the second intervention, a 1-month follow-up was also done. Finally, we met MD and his spouse 8 months after the end of the second intervention for a follow-up interview.

\section{First intervention: Emotion recognition and expression training}

The main objectives of the first intervention were to train MD to focus on relevant facial features and to associate these features with basic emotions (anger, joy, surprise, sadness, disgust, fear), as well as to increase awareness of his own emotions through imitation and introspection so that his own emotional experience could help him to identify others' feelings (Radice-Neumann et al., 2009; Neumann et al., 2015). Four main learning strategies were used: vanishing cues (i.e., cues that guide MD's attention toward relevant features of the faces), repetition, imitation and feedback. Modelling was also used in that, before each exercise, the therapist demonstrated how to do it.

This programme, adapted from "Faces Intervention" (Radice-Neumann et al., 2009; Neumann et al., 2015), is a computer-based programme of emotional faces (NimStim Faces; Tottenham et al., 2009) that vary in gender and race and express the six basic emotions. The programme contains six modules: (1) psychoeducation, (2) emotion identification training with vanishing cues and mirror use for imitation, (3) similarity/discrimination exercises, (4) expression and recognition of internal signals (body reactions) related to each basic emotion and deep understanding of emotions, (5) 
emotion attribution exercises and role playing, and (6) recapitulation (see Appendix A for a description of each module).

\section{Second intervention: the self-regulation programme}

The main objective of the second intervention was to provide MD with an alternative adapted behaviour in at-risk situations. This intervention contained four modules: (1) psychoeducation and identification of self-control strategies (e.g., behaviour interruption, putting in perspective, suppression, distraction, relaxation), assertiveness training (Nezu et al., 1991) and brief relaxation training with deep breathing (Sessions 1-2); (2) development of an alternative assertive behaviour to anger outbursts derived from the first module: the "PREP" acronym, representing four steps (stop, breath, assess anger, share emotion or disagreement peacefully with others) toward an adequate and assertive behaviour that could be easily encoded and remembered by using spaced retrieval (Sessions 2-3); (3) implementation intentions: general definition, training the "if-then" contingency through fictive scenario analysis and imagery training (adapted from Burkard, Rochat, Blum, et al., 2014) (Sessions 3-4); and (4) application of the "PREP" acronym in daily life by using implementation intentions (Sessions 5-8) (see Appendix B for a description of each module).

As a classic "stop-think-do strategy", the implementation intentions used in the current study included a "stop" component that aims to prevent the person from acting impulsively. However, the stop-think-do strategy and the implementation intentions strategy differ inasmuch as the latter constitutes a self-regulation strategy that automatically triggers the initiation of a specific action in a specific targeted situation (as defined in the "If..., then..." contingency), without the person deliberating on when to act, whether or not to act, or how to act. More specifically, implementation intentions 
were used in the current study to automatically initiate the previously established assertive behaviours in specific targeted situations of the patient's daily life. By contrast, the stop-think-do strategy refers to a self-regulation strategy used as a contentfree 'meta-prompt' to alert the person to engage in a problem resolution process (e.g., Nott et al., 2008).

\section{Statistical analyses}

The analysis of single-case data is still a controversial topic and no consensus has been reached (Kratochwill et al., 2010; Smith, 2012). Thus, we decided to follow the indications of Item 13 of the RoBiNT methodological quality scale (Tate et al., 2013), which promotes using visual analysis, aided by quasi-statistical techniques and welljustified statistical procedures. As a quasi-statistical procedure, the non-overlap of all pairs (NAP; Parker \& Vannest, 2009) focuses on data overlap (i.e., what proportion of data points improves the measurements from the previous phase), with values between 0 and 0.66 indicating a small effect size, and those between 0.93 and 1.00 indicating a large effect size. The slope and level change (SLC; Solanas, Manolov, \& Onghena, 2010) quantifies progressive change (if present) and average change in level (useful when the effect is abrupt and sustained). Unlike NAP, which yields a proportion, the SLC quantifications are provided in the same measurement units as the dependent variable (in this case, the number and intensity of anger outbursts). Finally, statistical analysis was performed by using a variant of Cohen's $d$ specifically adapted for singlecase designs to provide a broader quantitative summary of the results considering both interventions jointly. Specifically, we used the multiple-baseline version of the $d$ statistic (Hedges, Pustejovsky, \& Shadish, 2013), comparing the initial baseline phase (A) to the intervention phases (B or C). The $d$-statistic was obtained by using the $\mathrm{R}$ code (R Core Team, 2013) from Shadish, Hedges and Pustejovsky (2014). 


\section{RESULTS}

\section{Intervention 1: Emotion recognition and expression programme}

Figure 2 (upper panels) represents the measurements of frequency and median intensity of anger outbursts in the context of studying the effect of Intervention 1 . The visual analysis indicates that the initial baselines are stable, allowing comparisons with the subsequent intervention condition. In addition, the introduction of the intervention is associated with a delayed change in level for frequency and a clearer change in level for intensity. At the post-test, an increase in the frequency of anger outbursts can be observed, whereas the data for intensity suggest maintenance of the effect with respect to the intervention phase.

Regarding data overlap (Table 2), the NAP indicates that a greater reduction took place for anger intensity: $93 \%$ of the intervention phase values are smaller than the preintervention values, which corresponds to a large effect. For frequency, the value of NAP is indicative of a lack of effect (Vannest \& Ninci, 2015). The SLC quantifications provided in Table 2 show that when the intervention was introduced, there was an average decrease of 1.5 anger outbursts per measurement occasion (i.e., per week), although the net level change is close to 0 after this progressive change is taken into account. The evidence about median intensity is mixed, as the changes in level and in slope are in opposite directions. However, the results of SLC for intensity need to be validated by visual analysis (see Parker, Cryer, \& Byrns, 2006). More specifically, the decrease in level is visually clear, but the there are no visually clear trends in either the A or the B phase and thus the slope change estimate for this comparison seems less relevant. For the post-tests (i.e., the BM comparisons; see Table 2), an increase in outburst frequency was observed (i.e., a change in level of 6.98), but the slope change 
$(-0.48)$ implies a progressive reduction. For intensity, the low value of NAP reflects the fact that the post-test values are never higher than the intervention phase values. The effect is thus maintained.

\section{--INSERT FIGURE 2 ABOUT HERE-}

\section{--INSERT TABLE 2 ABOUT HERE--}

\section{Intervention 2: Self-regulation programme}

Figure 2 (lower panels) represents the measurements of frequency and median intensity of anger outbursts in the context of studying the effect of Intervention 2. First, baseline data for frequency and intensity are stable, allowing comparisons with the subsequent intervention condition. The introduction of the self-regulation intervention is associated with a progressive reduction of frequency and a more immediate improvement in intensity, which is, however, not maintained until the end of the intervention phase. The post-test (maintenance) data are variable and no firm conclusions can be drawn, although there is apparently a deteriorating trend for the frequency data. The follow-up data indicate that the results obtained during the intervention phase are maintained in terms of frequency and improved in terms of intensity.

Regarding data overlap (Table 2), the NAP indicates that when the self-regulation programme was introduced, there was a medium-sized reduction in frequency (approximately $90 \%$ of the baseline sessions included more frequent outbursts than the intervention phase sessions) and in intensity (rate higher for $83 \%$ of the pre-intervention sessions). The SLC quantifications provided in Table 2 show that when the intervention was introduced, there was a reduction in frequency of anger outbursts: this progressive decrease was less than half an outburst per week $(-0.43)$, plus an average decrease of 
almost one outburst $(-0.88)$ after the progressive change was taken into account. The evidence about median intensity is mixed, as the change in level implies an overall reduction, but there is an upward (i.e., deteriorating) trend. For the post-tests (i.e., the CM comparisons; see Table 2), there is an increasing trend in terms of frequency (0.43), whereas the frequency in the follow-up phase is identical to that in the maintenance phase. For intensity, the CM comparison indicates a decrease $(-1.12)$, which is also the case when comparing the maintenance phase with the subsequent follow-up $(-1.50)$.

However, slopes are not to be interpreted when only two values per phase are available.

In general, in quantitative terms, the intervention that proved more effective was the self-regulation programme, and its effect was larger for the frequency of outbursts than for their intensity.

Regarding perceived physical fatigue, the data (see Figure 3, Appendix C) suggest that no changes took place with the introduction of the interventions for this variable. However, there was a clear decrease of perceived fatigue from Intervention 1 to Intervention 2 (approximately $50 \%$ for both frequency and intensity of the target behaviour), which was likely due to the introduction of the positive airway pressure device between the two interventions.

\section{$d$-statistic}

The $d$-statistic shows that there was an improvement associated with the two interventions, both in terms of frequency and intensity: an average reduction of 0.46 standard deviations for frequency $(\mathrm{SE}=0.53,95 \%$ confidence interval ranging from -1.50 to 0.58$)$ and 0.12 standard deviations for intensity $(\mathrm{SE}=2.90,95 \%$ confidence interval ranging from -5.80 to 5.56). However, in both cases, the confidence intervals include 0 and thus we cannot state that the results are statistically significant. 
Nevertheless, in single-case data, statistical inference is not common and not justified when referring to a population of individuals. Combining both results meta-analytically, the overall result ( $d=0.46,95 \%$ confidence interval ranging from -1.47 to 0.57 ) is more heavily determined by the effect size for the frequency data for which the variance of the indicator is smaller and the confidence interval narrower. A value of 0.46 can be interpreted as a medium effect size using Cohen's (1992) criteria. Note that Shadish, Hedges, Horner and Odom (2015) recommended to perform such $d$-statistic on at least three cases which implies that the results reported here should be interpreted with caution.

\section{Tasks and questionnaires}

\section{Emotion recognition}

MD had difficulties in recognising fear (10\% to $40 \%$ of correct responses) and to a lesser extent anger (50\% of correct responses), sadness (from $50 \%$ to $60 \%$ of correct responses) and disgust (from $50 \%$ to $80 \%$ of correct responses) from static faces on the three baseline measurements (Table 3). MD often confounded surprise and fear, anger and sadness, or sadness and disgust.

\section{--INSERT TABLE 3 ABOUT HERE--}

The eye-tracker data on the third baseline underlines that MD focused much more on the mouth than on the area around the eyes when making a judgment. After Intervention 1, not only did his rate of correct responses dramatically increase (from $65 \%$ to $92 \%$ of correct responses), but his performances also generalised to stimuli taken from another battery, the $\mathrm{KDEF}$ ( $85 \%$ of correct responses). In addition, as expected, the eye-tracker data revealed that after the intervention, MD's gaze fixations were much more optimally distributed between the mouth and the area around the eyes 
than they were before the intervention (Table 4). Finally, emotion recognition from the voice (Table 5) did not improve after the intervention. Altogether, these data indicate that the intervention strategy consisting of orienting MD's attention to relevant features of the faces had positive and specific effects on emotion recognition from static faces.

\section{--INSERT TABLES 4 AND 5 ABOUT HERE--}

Regarding the questionnaires completed by MD's spouse (Table 6), a slight decrease was observed on the NPI after the first intervention on the agitation/aggression, anxiety, and disinhibition/impulsivity subscales. After the second intervention, symptoms of agitation/aggression continued to decrease, as did irritability.

\section{--INSERT TABLES 6 ABOUT HERE--}

On the DEX, a slight decrease was observed on the behaviour subscale (e.g., "He loses his temper at the slightest thing"; "He does or says embarrassing things when in the company of others"), especially after the second intervention. Finally, there was no improvement on the UPPS Impulsive Behaviour scale, which remained stable after the two interventions.

\section{Qualitative data}

After the first intervention, MD reported that he gained some insight into his own functioning and that from now on he could better understand his own emotional reactions. However, he described understanding what others are feeling as remaining difficult, especially during stressful interactions "because everything goes so fast that it is difficult to prevent myself from overreacting". His general satisfaction with the intervention was low to moderate (6 of 10). These remarks were corroborated by his wife. Her general satisfaction with the intervention was moderate (7 of 10). 
During the second intervention, MD's remarks on the use of implementation intentions indicated that they are easy to use. He also acknowledged that the "stop and breathe" portion of the PREP constituted the basis of the strategy and that it was very helpful for him, which increased his motivation to use the strategy. During the intervention, he reported using the strategy four to six times a week and completing the whole PREP process on two of three occasions. He also described a spontaneous use of the strategy in a situation that he had not been directly trained for during the intervention (e.g., "If my daughter responds to me badly, then I do the PREP"). In that case, only the verbal part of the intentions was used because he had no time to imagine himself performing an adapted behaviour in that situation. However, in cases of failure (i.e., when an anger outburst occurred), he could not explain why the strategy failed. When specifically questioned, he hypothesised that situations that were too arousing or that arose when he was experiencing excessive fatigue may compromise its effectiveness. After the intervention, he reported being proud to be able to express his disagreement with his children in a more adapted way. His general satisfaction with the intervention was moderate to high ( 8 of 10). His wife observed the use of PREP during stressful situations, as she noted that he tended to stop and breathe deeply before reacting. She also reported that he was less stressed during potential conflictual situations. Her general satisfaction with the intervention was moderate to high (8 of 10).

At the 8-month follow-up, both MD and his wife reported less frequent anger outbursts (either none or one, rarely two episodes per week) and better relationships with his children. His wife also noticed that he was more concerned about the children (e.g., he asked her questions about their children's activities more frequently) and he became more concerned about her when she had difficulties in her job. However, MD reported that he no longer used the implementation intentions in their entire form, but 
still used the "stop and breathe" part to prevent himself from (re)acting impulsively one or two times a week.

\section{DISCUSSION}

The patient described in this study sustained a severe TBI with persisting anger outbursts. These anger outbursts were very disturbing to MD and his family and he was motivated to benefit from an intervention that aimed to decrease both the frequency and the intensity of the anger outbursts. Consequently, we more specifically examined the efficacy of two successive and complementary interventions, namely a programme designed to improve emotion expression and recognition (Intervention 1) and a selfregulation programme including various features (psychoeducation, relaxation and assertiveness training) supported by implementation intentions (Intervention 2).

Globally, the results upheld the efficacy of these two interventions for decreasing the frequency and the intensity of anger outbursts. To the best of our knowledge, no previous single-case study has examined the effectiveness of these types of interventions designed to decrease anger outbursts after a TBI.

Regarding the first intervention, the results indicate that MD improved his ability to decode emotions from static faces after the intervention. These results corroborate previous data demonstrating an increase in emotion recognition performances in patients with TBI following an analogue intervention (Radice-Neumann et al., 2009; Neumann et al., 2015). Of note, the results also speak in favour of a specificity of the effect of the intervention. Indeed, emotion recognition improved for static faces, whereas no improvement occurred for voices. In addition, the pattern of visual exploration of the faces after the intervention indicates a better allocation of gaze fixations on relevant features of the face (eyes and mouth) than was the case before the 
intervention. This result was expected, considering the strategy used during the intervention, namely focusing attention on the relevant part of the face by using vanishing cues. Furthermore, the results generalised to a battery of stimuli (KDEF) that had not been presented before. Consequently, the improved performance in emotion recognition on the Ekman face battery observed after the intervention cannot be accounted for by multiple presentations of these stimuli.

Regarding the main outcomes, the results of the first intervention showed a decrease in the intensity of anger outbursts, indicating a transfer of learning to everyday life. This result is also supported by the decrease of disinhibition, irritability and aggression/agitation symptoms reported by the spouse on the questionnaires after the intervention. An interpretation of the positive effects of this intervention may be that this better ability to decode emotions from faces enables MD to better adjust his behaviours in relation to the emotions expressed by his interlocutors (e.g., to avoid becoming confrontational if the interlocutor expresses sadness or to discontinue behaving aggressively if the interlocutor displays fear).

However, the frequency of anger outbursts tended to increase at the post-test. One potential explanation for this increase lies in the fact that MD showed elevated fatigue before the introduction of the positive airway pressure device between the two interventions, which may have prevented him from fully benefitting from the first intervention. More generally, further studies should examine whether the benefits of the intervention in terms of frequency and/or intensity of anger outbursts could be improved and maintained in the long term by, for instance, practising emotion recognition with family members, which could help to boost or extend the transfer of learning in daily life. 
Regarding the second intervention, the results indicate that the frequency of anger outbursts slightly decreased after the intervention, but then increased at the post-test, whereas intensity continuously decreased during intervention, at the post-test and at follow-up. These results are also supported by the decrease in symptoms of disinhibition, irritability and aggression/agitation reported by MD's spouse on the questionnaires after the intervention.

At a qualitative level, MD considered the PREP strategy easy to use and said that stopping before overreacting tended to become a good habit. In addition, he reported being proud to be able to express his disagreement with his children in a more adapted way. One explanation for MD's positive reactions to the intervention may be that it increased his sense of control over his internal and/or external environment and that this feeling of mastery in turn improved his self-concept. Furthermore, according to his spouse, MD stopped, tried to cool down and seemed less distressed in at-risk situations, which speaks in favour of the use of the strategy in his daily life, although in the long term, MD no longer used the strategy that he was trained in during the intervention.

Overall, the results of this second intervention showed that a multicomponential self-regulation programme that includes implementation intentions to facilitate transfer of learning in the patient's daily life constitutes a relevant strategy to increase selfcontrol in a patient with a severe TBI. They also converge with previous data (e.g., Gollwitzer, 1999), indicating that the efficacy of implementation intentions principally depends on automatic, involuntary processes, as MD had moderate impairments in various laboratory measures of executive functioning, yet benefited from the strategy. In addition, in contrast with the findings of Churchill and Jessop (2010, 2011), who demonstrated that participants with a high level of urgency (defined as the tendency to experience strong reactions in emotional contexts) did not benefit from implementation 
intentions, our data corroborate Burkard et al.'s (2013) results that showed that this selfregulation strategy could be efficacious in persons with an elevated level of urgency. This result is of particular importance because urgency has been strongly associated with problematic behaviours, poor functional outcome and caregivers' burden after a TBI (Rebetez, Rochat, Ghisletta, Walder, \& Van der Linden, 2015; Rochat, Beni, Billieux, Annoni, \& Van der Linden, 2011). The fact that MD remained as impulsive on the UPPS Impulsive Behaviour scale at pre- and post-interventions suggests that both intervention strategies provided MD with knowledge and strategies to better control his behaviours in at-risk situations, rather than decreasing impulsivity as a trait per se.

The treatment for sleep apnoea by the positive airway pressure device and the resulting improvements in the patient's symptoms of fatigue contributed to the decrease of both frequency and intensity of anger outbursts in between the two interventions, which might be explained by an increase of cognitive resources available for selfcontrol. In turn, it could explain, at least partially, why the second intervention was more effective in that the patient was more focused during the second intervention than during the first. More generally, the extent to which the second intervention benefited from the first remains unclear. Indeed, the combined effect of the two interventions may have also led to an incremental improvement in the patient's ability to control his behaviour.

Another feature of the data deserves discussion: the effect of the interventions on the frequency of anger outbursts is not immediate but delayed. Although we cannot rule out that extraneous variables might explain this decrease because of the quasi experimental design of the study, one cannot reasonably expect that the psychological interventions used in the current study have an immediate effect on the target behaviour. Indeed, the strategies had to be first discussed and learned before being applied in the 
patient's everyday life, which might account for the delay observed in some of the results.

Some limitations to the study must also be discussed. First, the AB design reduced the opportunity to gather evidence of experimental control over the target behaviours as a result of the interventions, inasmuch as uncontrolled factors might also account for the results (e.g., passage of time, maturation or environmental events; Tate et al., 2013). Further studies should thus use an experimental design (e.g., a multiple baseline design across participants) to increase internal validity. Second, the target behaviour was assessed with a home-made diary for which we could not provide any reliability data. Such a tool was used because, to the best of our knowledge, there is no available questionnaire or scale in French that specifically assesses the target behaviour. Third, regarding the specificity of the effect in the second intervention, we acknowledge that the number of features (relaxation, assertiveness training, discussion on emotion regulation strategies and an implementation intentions strategy) included in the programme make it difficult to appraise whether one of these features alone or their combination contributed to the results. Finally, although MD and his wife mainly attributed improvements to the stop and breathe strategy, there is no direct evidence that implementation intentions played a role in the use of this strategy inasmuch as just learning the stop and breathe strategy might be efficacious (i.e. learning of the PREP acronym). Consequently, further studies are needed to demonstrate the specific efficacy of implementation intentions to improve emotion regulation in patients with brain injury.

\section{Conclusion}


This study demonstrated the efficacy of two successive and complementary

psychological interventions, namely a programme to improve emotion recognition and expression and a self-regulation programme with various features, including implementation intentions to decrease the frequency and intensity of anger outbursts in a patient with a severe TBI. It remains to be determined whether the findings of the current study generalise to other patients experiencing long-standing anger outbursts or other socially maladapted behaviours. Future studies could also investigate whether the observed gains are sustained in the longer term following the training period, as well as ways to improve the efficacy of these interventions.

\section{Acknowledgments}

The authors declare no conflict of interest. 


\section{References}

Aboulafia-Brakha, T., Greber Buschbeck, C., Rochat, L., \& Annoni, J.-M. (2013).

Feasibility and initial efficacy of a cognitive-behavioural group programme for managing anger and aggressiveness after traumatic brain injury. Neuropsychological Rehabilitation, 23(2), 1-18.

Alderman, N. (2003). Contemporary approaches to the management of irritability and aggression following traumatic brain injury. Neuropsychological Rehabilitation, $13(1-2), 211-240$.

Babbage, D. R., Yim, J., Zupan, B., Neumann, D., Tomita, M. R., \& Willer, B. (2011). Meta-analysis of facial affect recognition difficulties after traumatic brain injury. Neuropsychology, 25(3), 277-285.

Baddeley, A. D., Emslie, H., \& Nimmo-Smith, I. (1994). The Doors and People Test: A test of visual and verbal recall and recognition. Bury St Edmunds, Suffolk: Thames Valley Test Company.

Baguley, I. J., Cooper, J., \& Felmingham, K. (2006). Aggressive behavior following traumatic brain injury: How common is common? Journal of Head Trauma Rehabilitation, 21(1), 45-56.

Belmont, A., Agar, N., \& Azouvi, P. (2009). Subjective fatigue, mental effort, and attention deficits after severe traumatic brain injury. Neurorehabilitation and Neural Repair, 23(9), 939-944.

Benton, A. L., Sivan, A. B., Hamisher, K. de S., Varney, N. R., \& Spreen, O. (1994). Contributions to neuropsychological assessment, a clinical manual (2nd ed). New York: Oxford University Press. 
Running head: REDUCING ANGER OUTBURSTS AFTER A TBI

Bornhofen, C., \& McDonald, S. (2008a). Emotion perception deficits following traumatic brain injury: A review of the evidence and rationale for intervention. Journal of the International Neuropsychological Society, 14(4), 511-525.

Bornhofen, C., \& McDonald, S. (2008b). Treating deficits in emotion perception following traumatic brain injury. Neuropsychological Rehabilitation, 18(1), 2244.

Brandstätter, V., Lengfelder, A., \& Gollwitzer, P. M. (2001). Implementation intentions and efficient action initiation. Journal of Personality and Social Psychology, 81(5), 946-960.

Brickenkamp, R., \& Zillmer, E. (1998). The d2 test of attention. Seattle, Washington: Hogrefe \& Huber Publishers.

Burkard, C., Rochat, L., Blum, A., Emmenegger, J., Juillerat Van der Linden, A.-C., \& Van der Linden, M. (2014). A daily-life-oriented intervention to improve prospective memory and goal-directed behaviour in ageing: A pilot study. Neuropsychological Rehabilitation, 24(2), 266-295.

Burkard, C., Rochat, L., \& Van der Linden, M. (2013). Enhancing inhibition: How impulsivity and emotional activation interact with different implementation intentions. Acta Psychologica, 144(2), 291-297.

Churchill, S., \& Jessop, D. (2010). Spontaneous implementation intentions and impulsivity: Can impulsivity moderate the effectiveness of planning strategies? British Journal of Health Psychology, 15(3), 529-541.

Churchill, S., \& Jessop, D. C. (2011). Too impulsive for implementation intentions? Evidence that impulsivity moderates the effectiveness of an implementation intention intervention. Psychology \& Health, 26(5), 517-530. 
Cohen, A. L., \& Gollwitzer, P. M. (2008). The cost of remembering to remember. In M. Kliegel, M. A. McDaniel, \& G. O. Einstein (Eds.), Prospective memory: Cognitive, neuroscience, developmental and applied perspectives (pp. 161-185). New York: Taylor \& Francis Group/Lawrence Erlbaum Associates.

Cohen, J. (1992). A power primer. Psychological Bulletin, 112(1), 155-159.

Cummings, J. L., Mega, M., Gray, K., Rosenberg-Thompson, S., Carusi, D. A., \& Gornbein, J. (1994). The Neuropsychiatric Inventory: Comprehensive assessment of psychopathology in dementia. Neurology, 44(12), 2308-2314.

D’Elia, L. F., Satz, P., Uchiyama, C. L., \& White, T. (1996). Color Trails Test. Professional manual. Odessa, FL: Psychological Assessment Resources.

Demark, J., \& Gemeinhardt, M. (2002). Anger and it's management for survivors of acquired brain injury. Brain Injury, 16(2), 91-108.

Driscoll, D. M., Dal Monte, O., \& Grafman, J. (2011). A need for improved training interventions for the remediation of impairments in social functioning following brain injury. Journal of Neurotrauma, 28(2), 319-326.

Ekman, P., \& Friesen, W. V. (1976). Pictures of facial affect. Palo Alto, CA: Consulting Psychologists Press.

Giles, G. M., \& Manchester, D. (2006). Two approaches to behavior disorder after traumatic brain injury. Journal of Head Trauma Rehabilitation, 21(2), 168-178.

Gollwitzer, P. M. (1993). Goal achievement: The role of intentions. European Review of Social Psychology, 4(1), 141-185.

Gollwitzer, P. M. (1999). Implementation intentions: Strong effects of simple plans. American Psychologist, 54(7), 493-503.

Gollwitzer, P. M. (2014). Weakness of the will: Is a quick fix possible? Motivation and Emotion, 38(3), 305-322. 
Running head: REDUCING ANGER OUTBURSTS AFTER A TBI

Gollwitzer, P. M., \& Sheeran, P. (2006). Implementation intentions and goal achievement: A meta-analysis of effects and processes. Advances in Experimental Social Psychology, 38, 69-119.

Hedges, L. V., Pustejovsky, J. E., \& Shadish, W. R. (2013). A standardized mean difference effect size for multiple baseline designs across individuals. Research Synthesis Methods, 4(4), 324-341.

Howard, D., \& Patterson, K. (1992). Pyramids and palm trees: A test of semantic access from pictures and words. Bury St Edmunds, Suffolk: Thames Valley Test Company.

Ietswaart, M., Milders, M., Crawford, J. R., Currie, D., \& Scott, C. L. (2008). Longitudinal aspects of emotion recognition in patients with traumatic brain injury. Neuropsychologia, 46(1), 148-159.

Kim, E., Lauterbach, E. C., Reeve, A., Arciniegas, D. B., Coburn, K. L., Scheier, M. F., ... Coffey, E. C. (2007). Neuropsychiatric complications of traumatic brain injury: A critical review of the literature (a report by the ANPA committee on research). Journal of Neuropsychiatry and Clinical Neuroscience, 19(2), 106127.

Kim, S. H., Manes, F., Kosier, T., Baruah, S., \& Robinson, R. G. (1999). Irritability following traumatic brain injury. Journal of Nervous and Mental Disease, 187(6), 327-335.

Knox, L., \& Douglas, J. (2009). Long-term ability to interpret facial expression after traumatic brain injury and its relation to social integration. Brain and Cognition, 69(2), 442-449.

Kratochwill, T. R., Hitchcock, J. H., Horner, R. H., Levin, J. R., Odom, S. L., Rindskopf, D. M., \& Shadish, W. R. (2010). Single case designs technical 
documentation. In What Works Clearinghouse: Procedures and standards handbook (Version 2.0). Retrieved from http://ies.ed.gov/ncee/wwc/pdf/reference_resources/wwc_scd.pdf

Lengfelder, A., \& Gollwitzer, P. M. (2001). Reflective and reflexive action control in patients with frontal brain lesions. Neuropsychology, 15(1), 80-100.

Lundqvist, D., Flykt, A., \& Öhman, A. (1998). The Karolinska Directed Emotional Faces-KDEF, CD ROM from Department of Clinical Neuroscience, Psychology section, Karolinska Institutet, ISBN 91-630-7164-9.

Mancuso, M., Magnani, N., Cantagallo, A., Rossi, G., Capitani, D., Galletti, V., ... Robertson, I. H. (2015). Emotion recognition impairment in traumatic brain injury compared with schizophrenia spectrum: similar deficits with different origins. The Journal of Nervous and Mental Disease, 203(2), 87-95.

McDaniel, M. A., Howard, D. C., \& Butler, K. M. (2008). Implementation intentions facilitate prospective memory under high attention demands. Memory \& Cognition, 36(4), 716-724.

McDonald, S., \& Saunders, J. C. (2005). Differential impairment in recognition of emotion across different media in people with severe traumatic brain injury. Journal of the International Neuropsychological Society, 11(4), 392-399.

Medd, J., \& Tate, R. L. (2000). Evaluation of an anger management therapy programme following acquired brain injury: A preliminary study. Neuropsychological Rehabilitation, 10(2), 185-201.

Milders, M., Ietswaart, M., Crawford, J. R., \& Currie, D. (2008). Social behavior following traumatic brain injury and its association with emotion recognition, understanding of intentions, and cognitive flexibility. Journal of the International Neuropsychological Society, 14(2), 318-326. 
Neumann, D., Babbage, D. R., Zupan, B., \& Willer, B. (2015). A randomized controlled trial of emotion recognition training after traumatic brain injury. Journal of Head Trauma Rehabilitation, 30(3), 12-23.

Nezu, C. M., Nezu, A. M., \& Arean, P. (1991). Assertiveness and problem-solving training for mildly mentally retarded persons with dual diagnoses. Research in Developmental Disabilities, 12(4), 371-386.

Nott, M. T., Chapparo, C., \& Heard, R. (2008). Effective occupational therapy intervention with adults demonstrating agitation during post-traumatic amnesia. Brain Injury, 22(9), 669-683.

Parker, R. I., Cryer, J., \& Byrns, G. (2006). Controlling baseline trend in single-case research. School Psychology Quarterly, 21(4), 418-443.

Parker, R. I., \& Vannest, K. (2009). An improved effect size for single-case research: Nonoverlap of all pairs. Behavior Therapy, 40(4), 357-367.

Radice-Neumann, D., Zupan, B., Tomita, M., \& Willer, B. (2009). Training emotional processing in persons with brain injury. Journal of Head Trauma Rehabilitation, $24(5), 313-323$.

R Core Team. (2013). R: A language and environment for statistical computing. Vienna: R Foundation for Statistical Computing. Retrieved from http:/www.Rproject.org/

Rebetez, M. M. L., Rochat, L., Ghisletta, P., Walder, B., \& Van der Linden, M. (2015). Association between impulsivity, emotional/behavioural hyperactivation and functional outcome one year after severe traumatic brain injury. Brain Injury, 29(10), 1175-1181. 
Rochat, L., Beni, C., Billieux, J., Annoni, J.-M., \& Van der Linden, M. (2011). How impulsivity relates to compulsive buying and the burden perceived by caregivers after moderate-to-severe traumatic brain injury. Psychopathology, 44, 154-168.

Rochat, L., Beni, C., Billieux, J., Azouvi, P., Annoni, J. M., \& Van der Linden, M. (2010). Assessment of impulsivity after moderate to severe traumatic brain injury. Neuropsychological Rehabilitation, 20(5), 778-797.

Scherer, K. R., \& Scherer, U. (2011). Assessing the ability to recognize facial and vocal expressions of emotion: Construction and validation of the emotion recognition index. Journal of Nonverbal Behavior, 35(4), 305-326.

Shadish, W. R., Hedges, L. V., \& Pustejovsky, J. E. (2014). Analysis and meta-analysis of single-case designs with a standardized mean difference statistic: A primer and applications. Journal of School Psychology, 52(2), 123-147.

Shadish, W. R., Hedges, L. V., Horner, R. H., \& Odom, S. L. (2015). The role of between-case effect size in conducting, interpreting, and summarizing singlecase research (NCER-2015-02). Washington, DC: National Center for Education Research, Institute of Education Sciences, U.S. Department of Education. Retrieved on October 12, 2016 from http://ies.ed.gov/ncser/pubs/2015002/

Smith, J. D. (2012). Single-case experimental designs: A systematic review of published research and current standards. Psychological Methods, 17(4), 510-550.

Solanas, A., Manolov, R., \& Onghena, P. (2010). Estimating slope and level change in $\mathrm{N}=1$ designs. Behavior Modification, 34(3), 195-218.

Spikman, J. M., Milders, M. V., Visser-Keizer, A. C., Westerhof-Evers, H. J., HerbenDekker, M., \& van der Naalt, J. (2013). Deficits in facial emotion recognition 
Running head: REDUCING ANGER OUTBURSTS AFTER A TBI

indicate behavioral changes and impaired self-awareness after moderate to severe traumatic brain injury. PloS One, 8(6), e65581.

Tateno, A., Jorge, R. E., \& Robinson, R. G. (2003). Clinical correlates of aggressive behavior after traumatic brain injury. Journal of Neuropsychiatry and Clinical Neurosciences, 15(2), 155-160.

Tate, R. L., Perdices, M., Rosenkoetter, U., Wakim, D., Godbee, K., Togher, L., \& McDonald, S. (2013). Revision of a method quality rating scale for single-case experimental designs and n-of-1 trials: The 15-item Risk of Bias in N-of-1 Trials (RoBiNT) Scale. Neuropsychological Rehabilitation, 23(5), 619-638.

Tottenham, N., Tanaka, J. W., Leon, A. C., McCarry, T., Nurse, M., Hare, T. A., ... Nelson, C. (2009). The NimStim set of facial expressions: Judgments from untrained research participants. Psychiatry Research, 168(3), 242-249.

Vannest, K. J., \& Ninci, J. (2015). Evaluating intervention effects in single-case research designs. Journal of Counseling \& Development, 93(4), 403-411.

Walker, A. J., Nott, M. T., Doyle, M., Onus, M., McCarthy, K., \& Baguley, I. J. (2010). Effectiveness of a group anger management programme after severe traumatic brain injury. Brain Injury, 24(3), 517-524.

Watts, A. J., \& Douglas, J. M. (2006). Interpreting facial expression and communication competence following severe traumatic brain injury. Aphasiology, 20(8), 707722.

Wechsler, D. (1997). Wechsler Adult Intelligence Scale-Third edition. San Antonio, TX: The Psychological Corporation.

Wieber, F., Thürmer, J. L., \& Gollwitzer, P. M. (2015). Promoting the translation of intentions into action by implementation intentions: Behavioral effects and physiological correlates. Frontiers in Human Neuroscience, 395. 
Wilson, B. A., Alderman, N., Burgess, P. W., Emslie, H., \& Evans, J. J. (1996).

Behavioural assessment of the dysexecutive syndrome. St Edmunds, UK:

Thames Valley Test Company.

Zimmermann, P., \& Fimm, B. (2005). Tests d'évaluation de l'attention. Würselen:

Psytest. 


\section{Appendix A}

\section{First intervention: Emotion recognition and expression programme}

The main objectives of the first intervention were to train MD to focus on relevant facial features and to associate these features with basic emotions (anger, joy, surprise, sadness, disgust, fear), as well as to increase awareness of his own emotions through imitation and introspection so that his own emotional experience could help him to identify others' feelings (Radice-Neumann et al., 2009; Neumann et al., 2015). Four main learning strategies were used: vanishing cues (i.e., cues that guide MD's attention toward relevant features of the faces), repetition, imitation and feedback. Modelling was also used in that, before each exercise, the therapist demonstrated how to do it.

The programme adapted from "Faces intervention" (Radice-Neumann et al., 2009; Neumann et al., 2015) is a computer-based programme of emotional faces (NimStim Faces; Tottenham et al., 2009) that vary in gender and race and that express the six basic emotions. The programme contains six modules:

(1) Psychoeducation. The goal of treatment and the importance of emotion expression and recognition for appropriate behaviours were discussed and basic emotions defined. Following Neumann et al. (2015), we described the purpose of the programme (to help MD better recognise how people feel), the importance of being able to recognise others' emotions in daily life (e.g., to more adequately interact with other people, to develop skills to be compassionate with others) and the importance of understanding one's own emotions to better recognise others' emotions.

(2) Emotion identification; brief description of emotional events; mirror use. This module aimed to train MD to focus on relevant features of faces to better recognise the expressed emotion. For each static face, MD was asked to select, from a list of the six 
basic emotions, the emotion that best described how the person was feeling. Visual (i.e., thin red circles) and verbal cues were initially used to highlight the relevant facial features, but these cues gradually vanished. After each response, feedback was provided. Following incorrect answers, all the verbal and visual cues that described the characteristics of the features and the emotion being expressed were displayed on the screen. Each training exercise was scored for the percentage of correct answers. Scores below $83 \%$ (e.g., $<20$ of 24 correct responses) required that the exercise be repeated. This threshold was used throughout the programme. There was no time limit and MD had to produce the right answer before proceeding to the next trial.

This module was divided into three parts. In the first exercise ("identify emotion"), MD was asked to correctly identify the emotion of each presented face among six labels. For the first 24 faces, both visual (red circles surrounding the mouth and the eyes) and verbal cues (a brief description of the main features of the face) were presented. Provided MD attained $>83 \%$ of correct responses, 24 additional faces were presented with the visual cues only and finally, 24 emotional faces were presented without any cues at all (vanishing cues). The second exercise ("identify emotion and describe personal experiences") followed the same structure, except that there were only 18 faces (six with both verbal and visual cues, six with only visual cues, six with no cues). For the last six faces, MD was also asked to briefly describe six personal events that matched each basic emotion. These descriptions were reused and more deeply analysed in Module 4 (see below). The third exercise ("identify emotion and imitate") included 12 faces and after each identification, MD was asked to imitate as precisely as possible the identified emotion on a small portable mirror. When necessary, the therapist encouraged and helped MD by giving him instructions to improve the quality 


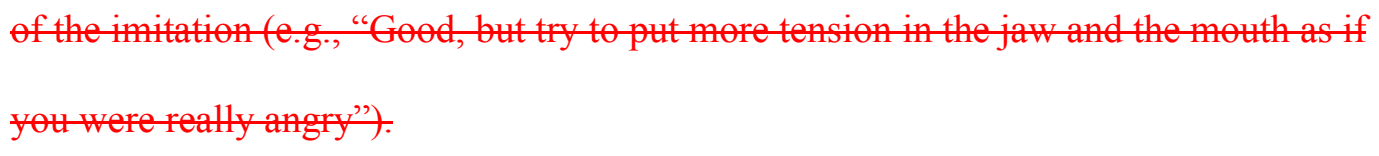

(3) Similarity/discrimination exercises. This module was divided into two parts. In the first series of exercises, MD was presented with two different faces (in terms of identity) at the same time and was asked to judge whether the faces expressed the same emotion or not. In the case of similarity, he was asked to correctly identify the emotion expressed by the faces. Visual cues were presented for the first block of 24 trials and vanished for the second block of 24 trials. After each correct response, MD had to justify his response (i.e., to provide a description of the features that were similar or dissimilar). In the second series of exercises, MD was shown five faces (one target and four distractors) at a time and was asked to identify, among the distractors, the one that matched the target in terms of emotion. Visual cues were presented for the first block of 24 trials and vanished for the second block of 24 trials. After each correct response, MD had to justify his response (i.e., to provide a description of the features that were similar between the target and the identified distractor). MD was then asked to name and imitate the emotion on the mirror.

\section{(4) Expression and recognition of internal signals (body reactions) related to each} basic emotion; deep understanding of emotions. In this module, MD was first asked to describe the physical and physiological changes of the body associated with each emotion (e.g., fear is associated with an increased heart rate, butterflies in the stomach). In the second part of the module, MD was asked to recall the personal events described in the first module as vividly as possible and was encouraged to feel what he "felt" at the time of the situation. He was asked to (a) describe the event in more detail, (b) describe his feelings and associated physiological reactions, (c) think about the reasons that he felt these emotions, (d) describe his response to the situation (both his 
behaviours and the emotional expression on his face and body), (e) think about others' perception of his emotional reactions and others' feelings, and (f) describe others' behaviour (including facial expression) and feelings in reaction to his own behaviour (for more details on this module, see Neumann et al., 2015). This exercise was repeated for each of the basic emotions and associated events described by MD in Module 1.

(5) Emotion attribution and role playing. In the first part of this module, MD saw a series of pictures presented one at a time depicting everyday life situations, with a short sentence describing the situation. For each situation, MD was asked to choose from among the six emotions represented by "emoticons" on the screen to indicate what he would have been feeling himself in this situation, to imitate this emotion and then to describe how another person (e.g., his children, his wife) would have been feeling in that situation. For instance, a picture depicts someone preparing a move with the following text: "Your son learned today that his best friend is moving to another country". Under the image, two sentences are displayed: "Your son feels__. With respect to your son, you feel ___ them, MD was the central character of the situation and was asked what he would have been feeling in that situation and then how his relative would react to his feelings; in the other half, as in the example above, he was asked to predict one of his relative's feelings and then his own feelings with respect to his relative. For each response, MD had to briefly justify his answers. In the second part of this module, MD was trained in emotion expression and recognition by role playing situations taken directly from his everyday life (e.g., MD meeting his son returning home too late on a Saturday night).

(6) Recapitulation. In this module, we reviewed with MD all the acquired competences learned in the previous modules. In addition, as he still tended to confuse one emotion with another (e.g., surprise vs. fear, disgust vs. anger) on some trials during the 
Running head: REDUCING ANGER OUTBURSTS AFTER A TBI

exercises, we presented each pair of confounded emotions on a screen and repeated the exercises by focusing MD's attention on the relevant part of the face and emphasising the differences between the features of each expressed emotion.

\section{Appendix B}

\section{Second intervention: The self-regulation programme}

The main objective of the second intervention was to provide MD with a strategy to better control his behaviour in at-risk situations. More specifically, by using various components (including intention implementations), we trained MD to stop emotional overreactions when they arise and to replace anger outbursts with appropriate assertive behaviours. This intervention contained four modules:

(1) Identification of self-control strategies and assertiveness (Session 1). In the first session, we discussed different self-control strategies (e.g., behaviour interruption, putting in perspective, suppression, distraction, relaxation) that were or were not used by MD and their possible applications, as well as their advantages and disadvantages.

MD used behaviour interruption (e.g., he left the place where the argument took place) most often as a way of coping with stressful situations. However, he felt annoyed and frustrated with this strategy because he could not share his emotion and communicate his disagreement with his interlocutors. Therefore, one objective of this second intervention was to provide a new strategy to cope with anger that might advantageously replace, when possible, behaviour interruption. In this context, an overall orientation to assertiveness (see Nezu, Nezu, \& Arean, 1991), including definitions and modelled examples of assertive, passive and aggressive behaviours, was presented and discussed with MD. At this point, MD was clearly able to acknowledge 
that his mode of communication was generally aggressive and sometimes passive, which prevented him from developing appropriate relationships with his children and provoked a lot of frustration.

Components of assertive behaviour were discussed with MD, including voice intensity (soft vs. loud), latency of response (appropriate vs. impulsive), eye contact (focused vs. unfocused), voice quality (assertive vs. angry), body language (appropriate vs. threatening) and listening ability (listening to the other person vs. talking constantly) (Nezu et al., 1991). Examples of various anger-provoking situations were presented, allowing the therapist to model assertive versus aggressive responses during role play. For example, in response to the situation in which "a neighbour falsely accuses you of throwing rubbish into his garden", MD was taught to respond only when the person had finished talking and not to interrupt, to use a calm voice, to not threaten the person, to look directly at the person when talking, to concretely and gently tell the person that he did not throw rubbish into his garden and to tell the neighbour how that accusation made him feel (e.g., angry, sad; see Nezu et al., 1991). The objective of this short assertiveness training was to build new interpersonal skills to better manage stressful situations associated with aggressive responding.

Brief relaxation training with deep breathing was also delivered for 5 to $10 \mathrm{~min}$ at the end of each session so that MD could experience the benefits of this technique to cool down. Relaxation training with deep breathing has been shown to improve cognitive functioning and to reduce stress after a traumatic brain injury (Hanna-Pladdy, Berry, Bennett, Phillips, \& Gouvier, 2001; Lysaght \& Bodenhamer, 1990) and was thus continuously used during the intervention.

(2) An alternative assertive behaviour to anger outbursts: "PREP" (Session 2). In this module, we proposed to MD an alternative behaviour to anger outbursts. More 
specifically, we elaborated the acronym PREP, representing four steps towards an adequate and assertive behaviour that could be easily encoded and remembered by using spaced retrieval. The four letters of the acronym were defined as follows (French/English):

$\mathbf{P}=$ Pause: Je m'arrête [Pause: I stop].

$\mathbf{R}=\mathbf{R e s p i r e r}$ profondément [Breathe deeply].

$\mathbf{E}=$ Evaluer ma colère sur un thermomètre allant de 1 "légère irritabilité" à 10 “agressivité avec contact physique". Si la colère $<3$, aller à l'étape 4 . Sinon, continuer à respirer ou quitter la pièce (interruption comportementale). [Assess my anger on a thermometer from 1 "slight irritability" to 10 "aggressive outburst with physical contact". If anger is $<3$, then go to Step 4 . If not, continue breathing or leave the place (behaviour interruption)]. $\mathbf{P}=$ Partager calmement mon émotion ou mon désaccord avec autrui [Share my emotion or my disagreement peacefully with others].

\section{(3) Implementation intentions: definition; training the "if-then" contingency} through scenario analysis and imagery training (adapted from Burkard, Rochat, Blum, et al., 2014) (Sessions 3-4). Implementation intentions (the "if-then strategy") were introduced and presented as a complementary self-regulation strategy to those already discussed in Module 1 (e.g., putting into perspective, relaxation, behaviour interruption). This psycho-educational part of the programme was illustrated with concrete, real-life examples.

After this introduction, the programme aimed to train MD in the verbal and then the visual components of implementation intentions separately, before training him to use combined verbal-visual implementation intentions. The verbal component was taught via scenarios in which a protagonist was in a stressful situation in which he might 
experience an anger outburst. Here, MD was trained to formulate a correct, precise "ifthen" sentence with the PREP acronym in the "then" component.

After MD was familiar with the verbal part of implementation intentions, he was trained in the visual component in parallel with the verbal component. We particularly insisted on the whole implementation intention being visualised; MD had to imagine himself performing the action in the situation, as in a short mental movie that had to be visualised from the actor's perspective rather than from the observer's perspective (Burkard, Rochat, Blum, et al., 2014). These mental movies can be multimodat (including visual or contextual elements, but also physiological reactions, others' remarks or behaviours, etc.). Visualisation exercises were then carried out. MD was invited to close his eyes and imagine a situation (e.g., the living room where he has frequent arguments with his children) in order to form a "mental movie". While imagining these situations, visualisation was fostered by asking questions about sensory and contextual information. Finally, he practised visualising implementation intentions (situational cue and to-be-performed behaviour). One central aspect of the programme was to define with MD specific, precise and relevant cues that trigger the action defined in the implementation intentions.

We also explained the difference between controlled and automatic processes and mentioned that implementation intentions relied principally on automatic processes. These explanations were proposed to prevent MD from believing that the technique worked by repeating the "if-then" sentences continuously until the appropriate time came for the intended behaviour to be performed (Burkard, Rochat, Blum et al., 2014).

\section{(4) Application of PREP in daily life by using implementation intentions (Sessions}

5-8). To allow the transfer to real-life situations, MD was prompted to give examples of his own anger outbursts and to observe which real-life situations would be appropriate 
as triggers for the "if" part of implementation intentions. Specific situations associated with frequent anger outbursts were practised during these sessions, and then used by MD at home.

We specifically focused on situations in which anger arises when MD had an argument with his children, as these situations were very painful to him. For instance, he had recurrent arguments with his son regarding the fact that his son wanted to replace some parts of his scooter with prohibited parts that make it dangerous to drive. Here, MD formulated an implementation intention ("If my son tells me that he wants to drive his scooter with non-approved parts, then I'll do the PREP"), and then repeated it three times. In addition, with the help of the therapist, MD formulated the alternative assertive behaviour to adopt in the last step of the "PREP" (e.g., "I gently tell him that I do not agree because it might be dangerous") before imagining himself acting in the situations and practising the intention.

When MD was familiar with the procedure, he was also prompted to include previously identified physiological signals (see Intervention 1, Module 4) associated with a rise in anger (e.g., warmth in the hand and cramps in the left leg) when formulating and then imagining the intention. In general, during each session, MD was trained in two specific implementation intentions. At the beginning of each session (Sessions 6 to 8), MD described whether he used the "if-then" strategy during the week, whether he used both verbal and visual components, and whether or not the implementation intentions were successful and the reason for potential failure. This information was discussed with the aim of optimising the use of implementation intentions in everyday life.

We also did an important check during each session: that MD was not too emotionally aroused before formulating the implementation intentions, as arousal 
constitutes a potential limit in the use of this strategy (Burkard et al., 2013). For this

purpose, we used a single item that assessed his arousal state on a 10-point Likert scale from 1 (very calm) to 10 (very excited). In each session, MD's score on this scale varied from 1 to 3, which seemed appropriate for the implementation intentions to be efficacious.

\section{Appendix C}

\section{Data on perceived fatigue}
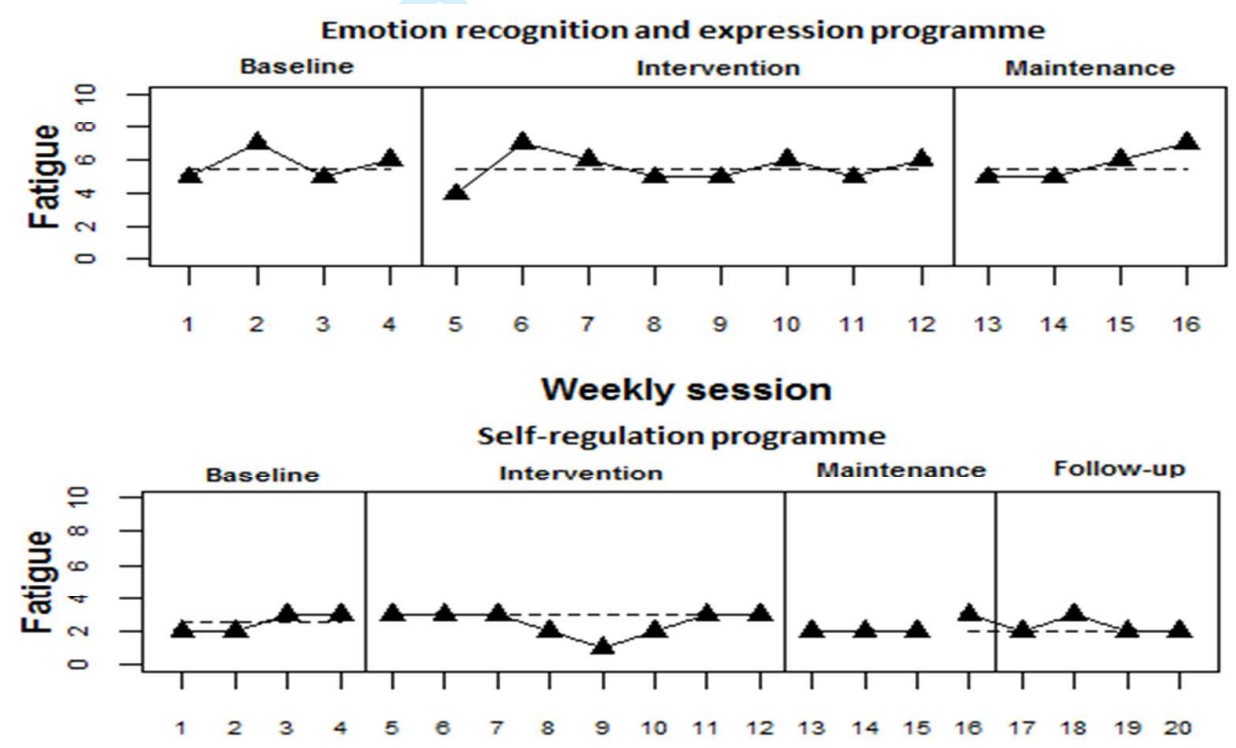

Weekly session 
Table 1. Neuropsychological assessment conducted with MD in March 2014

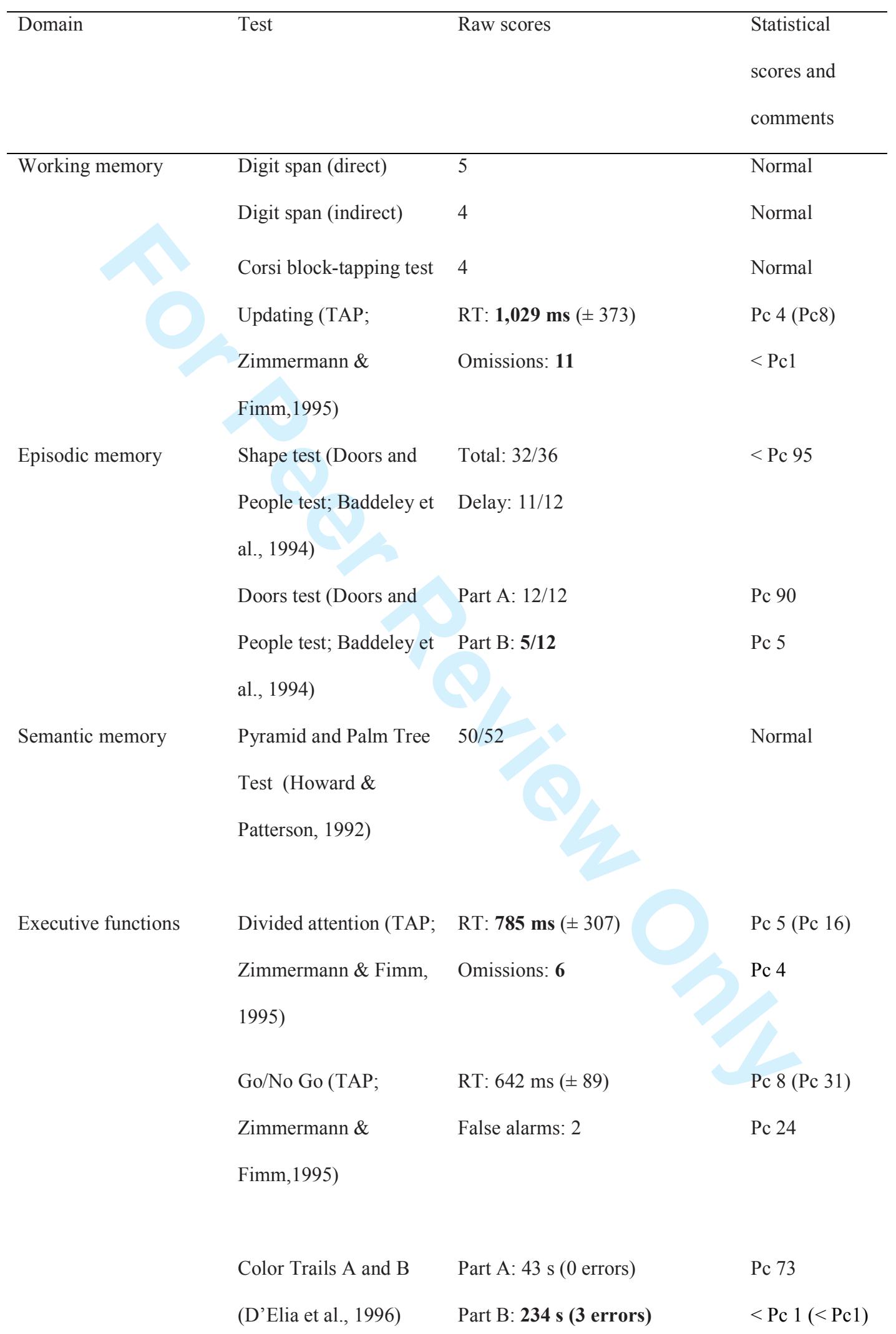


Non-verbal fluency (5- 24 productions, 1 perseveration Normal

point test)

Key test (BADS; $\quad$ Profile score: 1 Impaired

Wilson et al., 1996)

Attention

Alertness (TAP;

Without signal: $257 \mathrm{~ms}( \pm 31)$

$\operatorname{Pc} 31(\operatorname{Pc} 76)$

Zimmermann \&

With signal: $259 \mathrm{~ms}( \pm 50)$

Pc 24 (Pc 27)

Fimm,1995)

Index: -0.008

Pc 21

D2 (Brickenkamp \&

$\mathrm{GZ}=334$

Pc 8.1

Zillmer, 1998)

$\mathrm{F}=27$

$\mathrm{F} \%=8.08$

$\mathrm{GZ}-\mathrm{F}=307$

$\mathrm{SB}=15$

$\mathrm{KL}=114$

Code (WAIS-III;

36

Pc 25-50

Pc 11.5

Pc 25-50

Pc 21.2

Wechsler, 1997)

Face recognition

Benton Face

Normal

Recognition Test

(Benton et al., 1994)

Facial emotion

Ekman 60 Faces Test

Total: $39 / 60$

Surprise: $9 / 10$

(Ekman \& Friesen,

Sadness: $6 / 10$

Joy: $10 / 10$

1976)

Disgust: $8 / 10$

Fear: 1/10

Anger: 5/10

Std note $=5$ 
Table 2. Two-phase comparisons for the two types of intervention (emotion recognition and self-regulation) carried out using the non-overlap of all pairs and the slope and level change procedures

\begin{tabular}{|c|c|c|c|c|}
\hline \multicolumn{2}{|c|}{ Emotion recognition } & Non-overlap of all pairs & Slope change & Net level change \\
\hline \multirow{2}{*}{ Frequency } & $\mathrm{AB}$ & 59.37 & -1.52 & -0.04 \\
\hline & $\mathrm{BM}$ & 53.13 & -0.48 & 6.98 \\
\hline \multirow{2}{*}{ Intensity } & $\mathrm{AB}$ & 93.75 & 0.13 & -0.42 \\
\hline & $\mathrm{BM}$ & 10.00 & 0.20 & -0.30 \\
\hline \multicolumn{2}{|c|}{ Self-regulation } & Non-overlap of all pairs & Slope change & Net level change \\
\hline \multirow{3}{*}{ Frequency } & $\mathrm{AC}$ & 90.63 & -0.43 & -0.88 \\
\hline & $\mathrm{CM}$ & 29.69 & 0.43 & 0.80 \\
\hline & MF & 50.00 & 0.00 & 0.00 \\
\hline \multirow{3}{*}{ Intensity } & $\overline{\mathrm{AC}}$ & 83.33 & 0.20 & -1.08 \\
\hline & $\mathrm{CM}$ & 41.67 & 0.30 & -1.12 \\
\hline & MF & 12.50 & 0.00 & -1.50 \\
\hline
\end{tabular}

URL: http://mc.manuscriptcentral.com/pnrh Email: Duncan.nicholas@psypress.co.uk 
Table 3. Performances on emotion recognition task (Ekman faces) before and after Intervention 1

\begin{tabular}{|c|c|c|c|c|c|c|c|}
\hline & Happiness & Anger & Fear & Sadness & Surprise & Disgust & Total \\
\hline BL 1 & 100 & 50 & 10 & 60 & 90 & 80 & 65 \\
\hline BL 2 & 100 & 50 & 10 & 50 & 90 & 60 & 60 \\
\hline BL 3 & 100 & 50 & 40 & 60 & 90 & 50 & 65 \\
\hline Post-test & 100 & 90 & 90 & 90 & 90 & 90 & 92 \\
\hline KDEF & 100 & 90 & 80 & 70 & 80 & 90 & 85 \\
\hline
\end{tabular}


Table 4. Distribution of gaze fixation within eyes and mouth area

\begin{tabular}{lcc}
\hline & Eyes & Mouth \\
\hline BL 3 & 22.04 & 77.96 \\
Post-test & 42.59 & 57.41 \\
\hline Note. Performances are expressed as mean percentage of fixations related to total number of fixations \\
within the relevant area for all emotional categories. BL $=$ baseline.
\end{tabular}


Table 5. Performances on emotion recognition task (prosody) before and after Intervention 1

\begin{tabular}{lcccccc}
\hline & Happiness & Anger & Fear & Sadness & Neutral & Total \\
\hline BL 1 & 16.66 & 42.86 & 20 & 50 & 25 & 30.90 \\
BL 2 & 33.33 & 57.14 & 40 & 62.5 & 0.5 & 38.70 \\
BL 3 & 33.33 & 42.86 & 20 & 62.50 & 50 & 41.74 \\
Post-test & 16.66 & 42.86 & 20 & 50 & 50 & 35.90 \\
Note. Performances are expressed as percentage of correct responses. BL = baseline. &
\end{tabular}

Note. Performances are expressed as percentage of correct responses. $\mathrm{BL}=$ baseline.

URL: http://mc.manuscriptcentral.com/pnrh Email: Duncan.nicholas@psypress.co.uk 
Table 6. Scores on questionnaires before and after treatment for both interventions

\begin{tabular}{|c|c|c|c|c|c|c|c|}
\hline \multirow[b]{2}{*}{ Scales } & & \multicolumn{3}{|c|}{ Intervention 1} & \multicolumn{3}{|c|}{ Intervention 2} \\
\hline & & BL 1 & BL 2 & Post- & BL 1 & BL 2 & Post- \\
\hline \multirow[t]{7}{*}{ NPI } & Agitation/aggression & 12 & - & 9 & 8 & - & 4 \\
\hline & $\begin{array}{l}\text { Depressed } \\
\text { mood/dysphoria }\end{array}$ & 9 & - & 9 & 6 & - & 6 \\
\hline & Anxiety & 9 & - & 6 & 4 & - & 6 \\
\hline & Exaltation/euphoria & 0 & - & 1 & 0 & - & 0 \\
\hline & $\begin{array}{l}\text { Disinhibition/impulsivit } \\
\mathrm{y}\end{array}$ & 9 & - & 3 & 3 & - & 3 \\
\hline & Instability/irritability & 9 & - & 9 & 8 & - & 6 \\
\hline & Total & 48 & - & 37 & 29 & - & 25 \\
\hline \multirow[t]{4}{*}{ DEX } & Behaviour & 29 & 32 & 28 & 24 & 28 & 20 \\
\hline & Cognition & 7 & 6 & 7 & 8 & 7 & 6 \\
\hline & Emotion & 6 & 7 & 8 & 6 & 7 & 5 \\
\hline & Total & 42 & 45 & 43 & 38 & 42 & 31 \\
\hline \multirow[t]{4}{*}{ UPPS } & Urgency & 12 & 12 & 12 & 10 & 10 & 12 \\
\hline & Lack of premeditation & 12 & 14 & 12 & 10 & 8 & 12 \\
\hline & Lack of perseverance & 14 & 14 & 14 & 10 & 8 & 10 \\
\hline & Sensation seeking & 5 & 4 & 4 & 4 & 4 & 5 \\
\hline
\end{tabular}


6

7

8

9

10

11

12

13

14

15

16

17

18

19

20

21

22

23

24

25

26

27

28

29

30

31

32

33

34

35

36

37

38

39

40

41

42

43

44

45

46

47

48

49

50

51

52

53

54

55

56

57

58

59

60

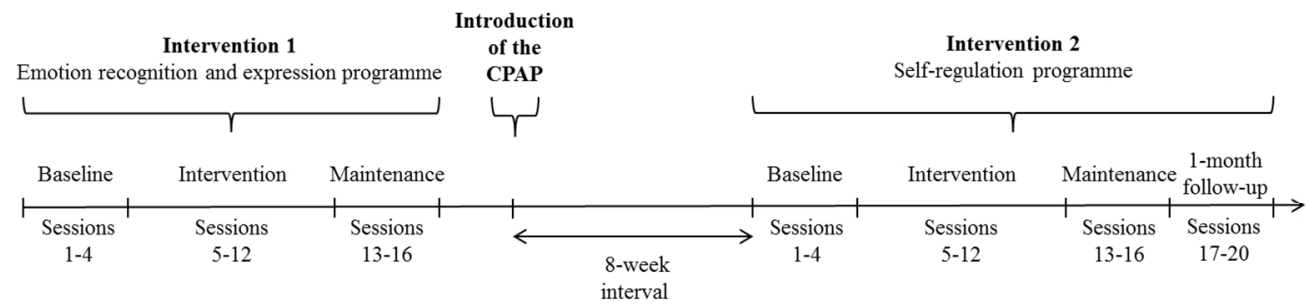

Figure 1. Schema representing the progress of the interventions. CPAP = continuous positive airway pressure. 
Emotion recognition

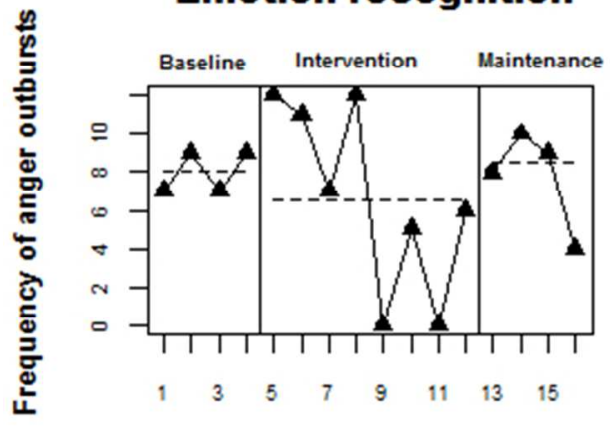

Weekly session

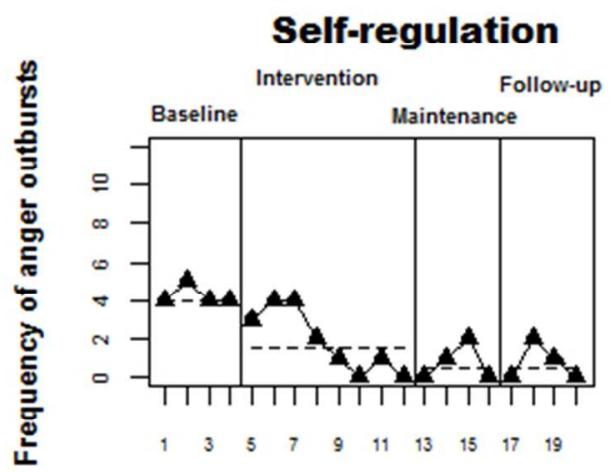

Weekly session

\section{Emotion recognition}

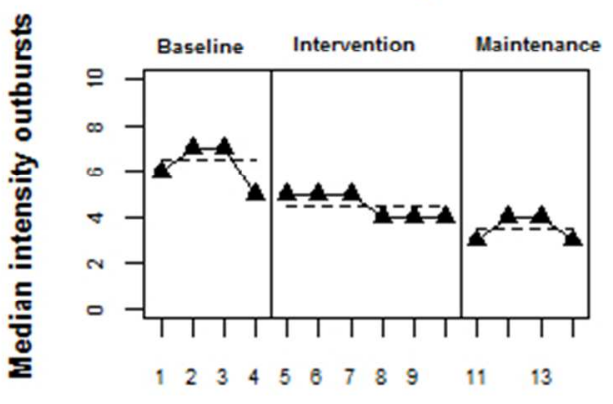

Weekly session

\section{Self-regulation}

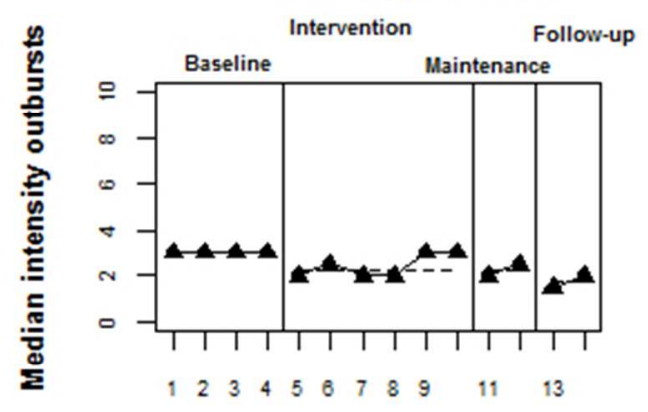

Weekly session

Graphical representation of the data obtained for frequency and intensity of anger outbursts under the two interventions. The dashed lines represent within-phase medians. 